\title{
Elliptic flow at collider energies and cascade string models: The role of hard processes and multi-Pomeron exchanges
}

\author{
E.E. Zabrodin ${ }^{a, b}$, C. Fuchs ${ }^{a}$, L.V. Bravina ${ }^{a, b}$, and Amand Faessler ${ }^{a}$ \\ ${ }^{a}$ Institute for Theoretical Physics, University of Tübingen, Auf der Morgenstelle \\ 14, D-72076 Tübingen, Germany \\ ${ }^{b}$ Institute for Nuclear Physics, Moscow State University, RU-119899 Moscow, \\ Russia
}

\begin{abstract}
Centrality, rapidity, and transverse momentum dependence of hadron elliptic flow is studied in $\mathrm{Au}+\mathrm{Au}$ collisions at BNL RHIC energies within the microscopic quarkgluon string model. The QGSM predictions coincide well with the experimental data at $\sqrt{s}=130 \mathrm{~A} \mathrm{GeV}$. Further investigations reveal that multi-Pomeron exchanges and hard gluon-gluon scattering in primary collisions, accompanied by the rescattering of hadrons in spatially anisotropic system, are the key processes needed for an adequate description of the data. These processes become essentially important for heavy-ion collisions at full RHIC energy $\sqrt{s}=200 \mathrm{~A} \mathrm{GeV}$.
\end{abstract}

PACS: 25.75.-q, 25.75.Ld, 24.10.Lx, 12.40.Nn

Key words: ultrarelativistic heavy-ion collisions, elliptic flow, hard and soft Pomeron, Monte-Carlo quark-gluon string model. 
One of the main goals of experiments on heavy-ion accelerators, including the Relativistic Heavy-Ion Collider (RHIC) at BNL, which is operating since 1999, and the coming Large Hadron Collider (LHC) at CERN, is to determine the equation of state (EOS) of nuclear matter under extreme conditions and to probe the deconfinement phase transition to the new state of matter dubbed quark-gluon plasma (QGP) (see, e.g., [1]). It has been understood long ago that nuclear matter expanding in the direction perpendicular to the beam axis of the colliding nuclei can carry information about the early stage of the reaction and subsequent evolution of the system [2]. This phenomenon known as "collective flow" has been intensively studied both theoretically and experimentally during the last two decades (see [3-5] and references therein). Nowadays the expansion in Fourier series is usually applied to study the azimuthal distribution of particles $[6,7]$ :

$$
E \frac{d^{3} N}{d^{3} p}=\frac{1}{\pi} \frac{d^{2} N}{d p_{t}^{2} d y}\left[1+\sum_{n=1}^{\infty} 2 v_{n} \cos (n \phi)\right],
$$

where $\phi$ is the azimuthal angle, and $p_{t}$ and $y$ is the transverse momentum and the rapidity of a particle, respectively. The first term in square brackets represents the isotropic radial flow, while the others are referred to anisotropic flow. The first Fourier coefficient $v_{1}=\langle\cos \phi\rangle \equiv\left\langle p_{x} / p_{t}\right\rangle$ is called directed flow, and the second one $\left.v_{2}=\cos (2 \phi)\right\rangle \equiv\left\langle\left(p_{x} / p_{t}\right)^{2}-\left(p_{y} / p_{t}\right)^{2}\right\rangle$ is dubbed elliptic flow. Here the beam axis is labelled as $z$-axis. Together with the impact parameter $x$-axis, it defines the reaction plane perpendicular to the $y$-axis. To study centrality, (pseudo)rapidity, and transverse momentum dependence of the anisotropic flow the following triple differential distributions integrated over any two of variables in given intervals are useful

$$
v_{n}\left(x_{i}\right)=\int_{x_{j}^{(1)}}^{x_{j}^{(2)}} \cos (n \phi) \frac{d^{3} N}{d x_{j}} d x_{j \neq i} / \int_{x_{j}^{(1)}}^{x_{j}^{(2)}} \frac{d^{3} N}{d x_{j}} d x_{j \neq i}
$$

where the variables $x_{j}, j=1,2,3$ denote the impact parameter $b$, transverse momentum $p_{t}$, and (pseudo)rapidity $(\eta) y$.

The importance of elliptic flow as a probe of the hot and dense nuclear phase at high energies has been discussed first in Ref. [8]. Both microscopic and macroscopic calculations seem to support the idea that elliptic flow is developed at the very early stage of a nuclear collision [9-15]. Several features, such as a characteristic "kinky" structure in the excitation function of elliptic flow [16], have been proposed to search for the QGP phase. The first data on elliptic flow of charged particles in the midrapidity range of $\mathrm{Au}+\mathrm{Au}$ collisions at $\sqrt{s}=130 \mathrm{~A} \mathrm{GeV}$, measured by the STAR Collaboration, became available recently [17]. It appears that the microscopic transport cascade models, e.g. 
ultra-relativistic quantum molecular dynamics (UrQMD) [18] or RQMD [16], predict too weak values of $v_{2}(b, \eta)[19,20]$, while hydrodynamic models overpredict the measured elliptic flow by about 20-50 \% [17]. In hydrodynamics the EOS can be softened by the introduction of a QGP phase and hadron resonance-rich matter $[21,22]$. In the present paper we demonstrate that the elliptic flow at RHIC can quantitatively be described within cascade string models by the mechanism of string excitations due to colour exchange. This procedure is different from the FRITIOF model of longitudinal excitations of strings [23] which is the basic part of the string sector in several ultrarelativistic transport models. For the further analysis the quark-gluon string model (QGSM) [24] is employed.

In the hadron-hadron $(h h)$ collision part of the QGSM statistical weights, hadron structure functions, and leading quark fragmentation functions have been obtained in Ref. [25] within the Gribov-Regge theory (GRT) [26]. This enables one to choose subprocesses of string excitations, calculate mass and momentum of a string, and simulate the string fragmentation into hadrons properly. Since a hadron is represented by a vector in the Fock space of constituents, namely valence and sea quarks, diquarks, gluons, and their antistates, the strings produced in hadronic collisions originate from different interactions between the constituents. Scattering of strings is approximated by scattering of the valence quarks and diquarks at the end of the strings, and of hadrons that are produced in string decays. Similar to the RQMD model the valence quarks and diquarks are allowed to interact promptly, while the newly produced hadrons can scatter only after a formation time related to the time needed to break a string. Together with subprocesses with quark annihilation and exchange associated with the Reggeon exchanges in GRT, the model includes subprocesses with colour exchange connected to one or more Pomeron exchanges in elastic amplitudes in the GRT. The hard gluon-gluon scattering with large momentum transfer and the so-called semihard processes with quark and gluon scattering are included in the QGSM as well [27]. The inelastic $h h$ cross section $\sigma_{i n}(s)$ can be calculated via the real part of the eikonal $u(s, b)$

$$
\sigma_{i n}(s)=2 \pi \int_{0}^{\infty}\left\{1-\exp \left[-2 u^{R}(s, b)\right]\right\} b d b
$$

Here $s$ is the center-of-mass energy of the reaction. The eikonal can be presented as a sum of three terms corresponding to soft and hard Pomeron exchange, and triple Pomeron exchange, which is responsible for the single diffraction process,

$$
u^{R}(s, b)=u_{\text {soft }}^{R}(s, b)+u_{\text {hard }}^{R}(s, b)+u_{\text {triple }}^{R}(s, b) .
$$


Using the Abramovskii-Gribov-Kancheli (AGK) cutting rules [28] the inelastic cross section of $h h$ interaction can be presented as

$$
\begin{aligned}
\sigma_{i n}(s) & =\sum_{i, j, k=0 ; i+j+k \geq 1} \sigma_{i j k}(s) \\
\sigma_{i j k}(s) & =2 \pi \int_{0}^{\infty} b d b \exp \left[-2 u^{R}(s, b)\right] \\
& \times \frac{\left[2 u_{\text {soft }}^{R}(s, b)\right]^{i}}{i !} \frac{\left[2 u_{\text {hard }}^{R}(s, b)\right]^{j}}{j !} \frac{\left[2 u_{\text {triple }}^{R}(s, b)\right]^{k}}{k !} .
\end{aligned}
$$

The last equation enables one to determine the number of cut soft and hard Pomerons, i.e., the number of strings and hard jets. The single Pomeron exchange, that can be represented by a cylinder-type diagram [25], leads to the formation of two quark-diquark or quark-antiquark strings. With rising energy the processes with multi-Pomeron exchanges become more and more important. The contribution of the cylinder diagrams to the scattering amplitude increases as $s^{\alpha_{P}(0)-1}$, while that of the so-called chain diagrams corresponding to $n$-Pomeron exchanges $(n \geq 2)$ rises as $s^{n\left[\alpha_{P}(0)-1\right]}$ with $\alpha_{P}(0)>1$ being the intercept of a Pomeranchuk pole. Diagrams corresponding to hard gluon-gluon scattering and double Pomeron exchange are shown as an example in Fig. 1(a) and (b), respectively. An overview and recent development of the GRT can be found in Ref. [29]. The cascade (rescattering of secondaries) is introduced in the QGSM as well. There are several other models which employ the colour exchange mechanism for string excitations, e.g., the dual parton model (DPM) [30] and the very energetic nuclear scattering model (VENUS) [31].

The centrality, transverse momentum, and rapidity dependence of anisotropic flow in the QGSM at energies from AGS to SPS has been studied in Refs. [3234]. Here we will focus on study of elliptic flow in minimum bias $\mathrm{Au}+\mathrm{Au}$ collisions at two energies, $\sqrt{s}=130 \mathrm{~A} \mathrm{GeV}$ and $200 \mathrm{~A} \mathrm{GeV}$, available at RHIC. Rapidity and pseudorapidity distributions of elliptic flow of pions and charges particles are depicted in Fig. 2. For both energies elliptic flow displays strong in-plane alignment in accordance with the predictions of Ref. [8]. In the $\operatorname{mid}($ pseudo)rapidity the flow is almost constant. At $\sqrt{s}=130 A \mathrm{GeV}$ it rises up slightly at $|y|,|\eta| \approx 1.5$, and then drops with the increasing rapidity. The mean value of the $v_{2}^{c h}(|\eta|<1.3)$ equals $4.16 \pm 0.5 \%$, that is very close to the value $4.5 \pm 0.4 \%$ measured by the STAR Collaboration [17]. Pseudorapidity dependence of the elliptic flow of charged particles in the whole $\eta$ range is also in a good agreement with the preliminary results reported by the PHOBOS Collaboration [35]. The QGSM predicts that at full RHIC energy elliptic flow of charged particles will increase further to $v_{2}^{c h}(|\eta|<1.3)=4.9 \pm 0.5 \%$. To elaborate on the influence of hard processes and multi-Pomeron exchanges on the elliptic flow formation the flow caused by the subprocesses without the 
hard and multichain contributions is also plotted in Fig. 2. It seems that in $\mathrm{Au}+\mathrm{Au}$ collisions at $\sqrt{s}=130 \mathrm{~A} \mathrm{GeV}$ the magnitude of the signal (except of the midrapidity range) can be reproduced without the many-string processes. At $\sqrt{s}=200 A \mathrm{GeV}$ their role becomes more significant, because the elliptic flow caused by other subprocesses cannot exceed the limit of $3-3.5 \%$. Here it is important to stress that the multichain diagrams alone, without rescattering, cannot affect the elliptic flow at all. The flow increases solely due to secondary interactions of produced particles in spatially asymmetric system.

Figure 3 presents the centrality dependence of elliptic flow of charged particles. Since the centrality of events in the experiment has been determined via the ratio of charged particle multiplicity to its maximum value $N_{c h} /\left(N_{c h}\right)^{\max }$, we compare the $v_{2}\left[N_{c h} /\left(N_{c h}\right)^{\max }\right]$ signal with the original impact parameter dependence $v_{2}(b)$. One can see that the ratio $N_{c h} /\left(N_{c h}\right)^{\max }$ is a good criterion of the event centrality except of the very central region with $b \leq 2.5 \mathrm{fm}$, where the multiplicity depends weakly on the impact parameter. However, as a function of the impact parameter $b$ elliptic flow is saturated at $b \approx 8 \mathrm{fm}$ for both energies, while as a function of the multiplicity ratio it increases nearly linearly with decreasing multiplicity up to $N_{c h} /\left(N_{c h}\right)^{\max } \approx 0.2$. As expected, the flow in the midrapidity region is caused mainly by pions. The magnitude of the pionic flow in the QGSM calculations is twice as large as obtained, e.g. with RQMD [20]. Without the many-string processes the QGSM is able to describe the flow only in central and semicentral collisions. It predicts a drop of the elliptic flow as the reaction becomes more peripheral, which is similar to the predictions of other string models $[19,20]$.

The difference in centrality dependences of elliptic flow in calculations with and without multi-string processes can be explained as follows 円. Hard jets and many-Pomeron exchanges produce more particles, thus giving rise to more rescatterings that drive the system toward thermal and chemical equilibrium. The centrality dependence of elliptic flow in this case is very close to that obtained at the hydrodynamic limit first in Ref. [8]. In contrast, the less number of produced secondaries would bring the system closer to the low density limit [14], which means incomplete (if any) thermalization of the system. In this regime elliptic flow in peripheral collisions vanishes much earlier compared to the hydrodynamic one $[14,36]$.

The transverse momentum dependence of elliptic flow is shown in Fig. 4. The agreement with the experimental data is good, although the model slightly overpredicts the elliptic flow of charged particles at $p_{t} \leq 0.5 \mathrm{GeV} / c$. Also, the dip in the excitation function of elliptic flow at $p_{t} \approx 1 \mathrm{GeV} / c$ looks peculiar. Nevertheless, both effects are attributed to the lack of heavy resonances in the model. This, however, cannot affect the rapidity and centrality distributions

$\overline{1}$ This point was clarified in discussions with J.-Y. Ollitrault 
where the spectra of particles are integrated over the whole transverse momentum range. Similar to the centrality dependence, the calculations without the many-string processes can explain the experimental $v_{2}\left(p_{t}\right)$ data only at $p_{t} \leq 0.8 \mathrm{GeV} / c$. To describe the further increase of elliptic flow with rising $p_{t}$ the full set of diagrams is required. At $p_{t} \geq 1.8 \mathrm{GeV} / c$ elliptic flow saturates in accord with experimental results [37]. Note that even without the many-string processes the elliptic flow of hadrons in the QGSM is stronger than that of the string models based on the FRITIOF routine. This is due to the fact that the string stretched between the constituents belonging to different hadrons is not parallel to the beam axis, thus giving the additional transverse push to secondaries.

In conclusion, the microscopic quark-gluon string model based on the colour exchange mechanism is able to reproduce quantitatively experimental data on elliptic flow in $\mathrm{Au}+\mathrm{Au}$ collisions at RHIC energies quite well, i.e. the microscopic models are still on the market for ultrarelativistic heavy-ion collisions. The key processes in the formation of strong elliptic flow at collider energies appear to be hard Pomeron exchange, resulting in the creation of hard partonic jets, and soft multi-Pomeron exchanges, accompanied by the subsequent rescattering of secondaries in spatially asymmetric system. Note, that directed and elliptic flow strongly depends on the mean free path of hadrons, which is inversely proportional to the particle density and interaction cross section. The larger the density (or cross section) of particles participating in secondary rescattering, the larger the elliptic flow [38]. Therefore, multi-Pomeron exchanges and hard processes increase the yield of secondaries at the initial stage of the reaction thus enhancing the elliptic flow. Without the many-string processes the model cannot describe the rise of elliptic flow $v_{2}(b)$ for peripheral events, while the magnitude of the flow $v_{2}^{\text {ch }}(y)$ in $\mathrm{Au}+\mathrm{Au}$ collisions at $130 \mathrm{~A}$ $\mathrm{GeV}$ and $200 \mathrm{~A} \mathrm{GeV}$ cannot exceed the limit of $3.5 \%$. For the $v_{2}\left(p_{t}\right)$ distribution the QGSM predicts the saturation of elliptic flow at $p_{t} \geq 1.8 \mathrm{GeV} / c$. This finding agrees quantitatively with the experimental results and qualitatively with the predictions of other string models, but deviates from the hydrodynamic calculations. More comparison is needed to distinguish unambiguously between hydro- and string- approaches, and to find clear signals of the quark-gluon plasma formation.

Acknowledgements. We are indebted to L. Csernai, J.-Y. Ollitrault, D. Strottman, V.D. Toneev, and S.A. Voloshin for interesting discussions and fruitful comments. This work was supported in part by the Bundesministerium für Bildung und Forschung (BMBF) under contract 06TÜ986, and by the Bergen Computational Physics Laboratory (BCPL) in the framework of the European Community - Access to Research Infrastructure action of the Improving Human Potential Programme. 


\section{References}

[1] Proceedings of the QM'99 conference [Nucl. Phys. A 661 (1999) 1c].

[2] W. Scheid, H. Müller, and W. Greiner, Phys. Rev. Lett. 32 (1974) 741.

[3] H. Stöcker and W. Greiner, Phys. Rep. 137 (1986) 277.

[4] W. Reisdorf and H.G. Ritter, Annu. Rev. Nucl. Part. Sci. 47 (1997) 663.

[5] N. Herrmann, J.P. Wessels, and T. Wienold, Annu. Rev. Nucl. Part. Sci. 49 (1999) 581.

[6] S. Voloshin and Y. Zhang, Z. Phys. C 70 (1996) 665.

[7] A.M. Poskanzer and S.A. Voloshin, Phys. Rev. C 58 (1998) 1671.

[8] J.-Y. Ollitrault, Phys. Rev. D 46 (1992) 229; Phys. Rev. D 48 (1993) 1132.

[9] H. Sorge, Phys. Lett. B 402 (1997) 251.

[10] J.-Y. Ollitrault, Nucl. Phys. A 638 (1998) 195c.

[11] H. Liu, S. Panitkin, and N. Xu, Phys. Rev. C 59 (1999) 348.

[12] B. Zhang, M. Gyulassy, and C.M. Ko, Phys. Lett. B 455 (1999) 45.

[13] P.F. Kolb, J. Sollfrank, and U. Heinz, Phys. Lett. B 459 (1999) 667.

[14] H. Heisenberg and A.-M. Levy, Phys. Rev. C 59 (1999) 2716.

[15] J. Brachmann et al., Phys. Rev. C 61 (2000) 024909.

[16] H. Sorge, Phys. Rev. Lett. 82 (1999) 2048; Nucl. Phys. A 661 (1999) 577c.

[17] STAR Collaboration, K.H. Ackermann et al., Phys. Rev. Lett. 86 (2001) 402.

[18] S.A. Bass et al., Prog. Part. Nucl. Phys. 41 (1998) 255; M. Bleicher et al., J. Phys. G 25 (1999) 1859.

[19] M. Bleicher and H. Stöcker, hep-ph/0006147.

[20] R.J.M. Snellings, A.M. Poskanzer, and S.A. Voloshin, STAR Note SN0388 (1999) (nucl-ex/9904003).

[21] D. Teaney, J. Lauret, and E.V. Shuryak, nucl-th/0011058.

[22] P.F. Kolb, P. Huovinen, U. Heinz, and H. Heiselberg, Phys. Lett. B 500 (2001) 232.

[23] B. Andersson, G. Gustafson, and B. Nilsson-Almquist, Nucl. Phys. B 281 (1987) 289.

[24] N.S. Amelin, L.V. Bravina, L.I. Sarycheva, and L.N. Smirnova, Sov. J. Nucl. Phys. 50 (1989) 1058; N.S. Amelin and L.V. Bravina, Sov. J. Nucl. Phys. 51 (1990) 133; N.S. Amelin et al., Phys. Rev. C 47 (1993) 2299. 
[25] A.B. Kaidalov, Sov. J. Nucl. Phys. 45 (1987) 902; Nucl. Phys. A 525 (1991) 39c; A.B. Kaidalov and K.A. Ter-Martirosyan, Sov. J. Nucl. Phys. 39 (1984) 979.

[26] V. Gribov, Sov. Phys. JETP 26 (1968) 414; L.V. Gribov, E.M. Levin, and M.G. Ryskin, Phys. Rep. 100 (1983) 1.

[27] N.S. Amelin, E.F. Staubo, and L.P. Csernai, Phys. Rev. D 46 (1992) 4873.

[28] V. Abramovskii, V. Gribov, and O. Kancheli, Sov. J. Nucl. Phys. 18 (1974) 308.

[29] H.J. Drescher, M. Hladik, S. Ostapchenko, T. Pierog, and K. Werner, hep$\mathrm{ph} / 0007198$.

[30] A. Capella, U. Sukhatme, C.I. Tan, and J. Tran Thanh Van, Phys. Rep. 236 (1994) 225.

[31] K. Werner, Phys. Rep. 232 (1993) 87.

[32] N.S. Amelin et al., Phys. Rev. Lett. 67 (1991) 1523.

[33] L.V. Bravina, Phys. Lett. B 344 (1995) 49.

[34] L.V. Bravina, E.E. Zabrodin, Amand Faessler, and C. Fuchs, Phys. Lett. B 470 (1999) 27; L.V. Bravina, Amand Faessler, C. Fuchs, and E.E. Zabrodin, Phys. Rev. C 61 (2000) 064902; E.E. Zabrodin, C. Fuchs, L.V. Bravina, and Amand Faessler, Phys. Rev. C 63 (2001) 034902.

[35] G. Roland for the PHOBOS Collaboration, Talk at the conference Quark Matter'2001 (January 15-22, Stony Brook, USA) (Nucl. Phys. A, in press).

[36] S.A. Voloshin and A.M. Poskanzer, Phys. Lett. B 474 (2000) 27.

[37] R.J.M. Snellings for the STAR Collaboration, nucl-ex/0104006 (Nucl. Phys. A, in press).

[38] D. Molnar and M. Gyulassy, nucl-th/0104018 (Nucl. Phys. A, in press). 
(a)

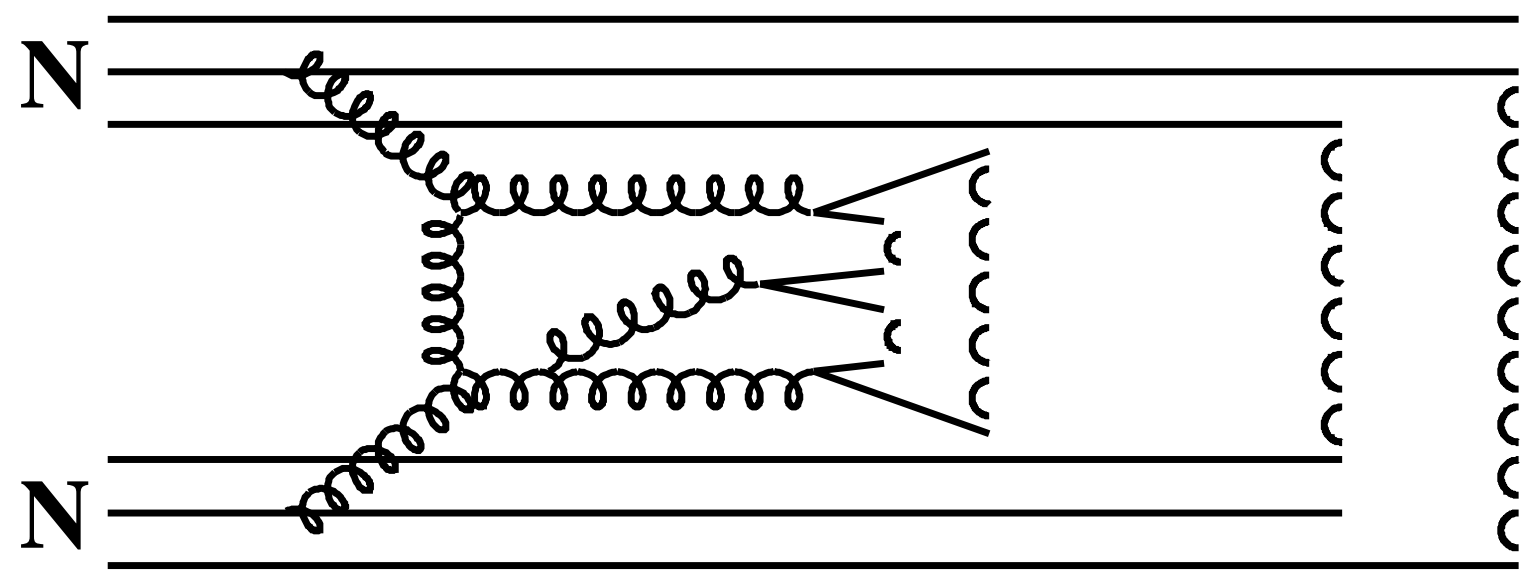

(b)

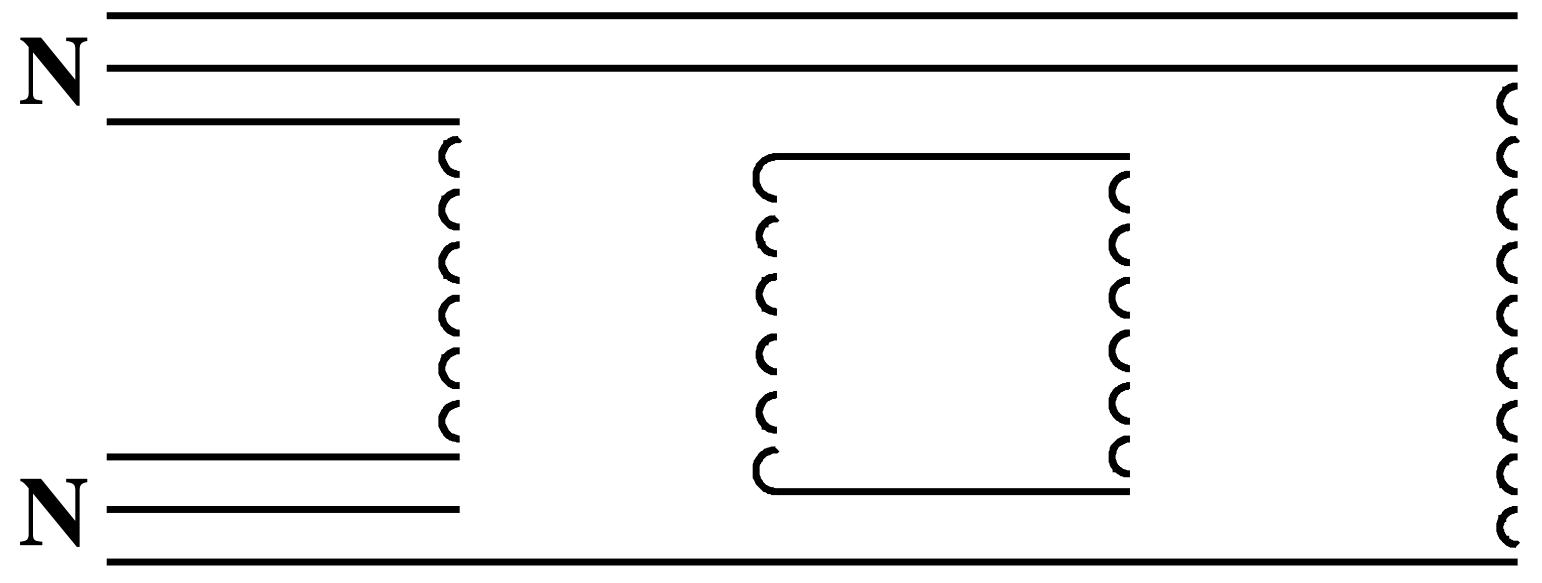

Fig. 1. (a) String formation in hard gluon-gluon scattering and soft Pomeron exchange in nucleon-nucleon (NN) collision. (b) Formation of four strings as a result of double Pomeron exchange in NN collision. 


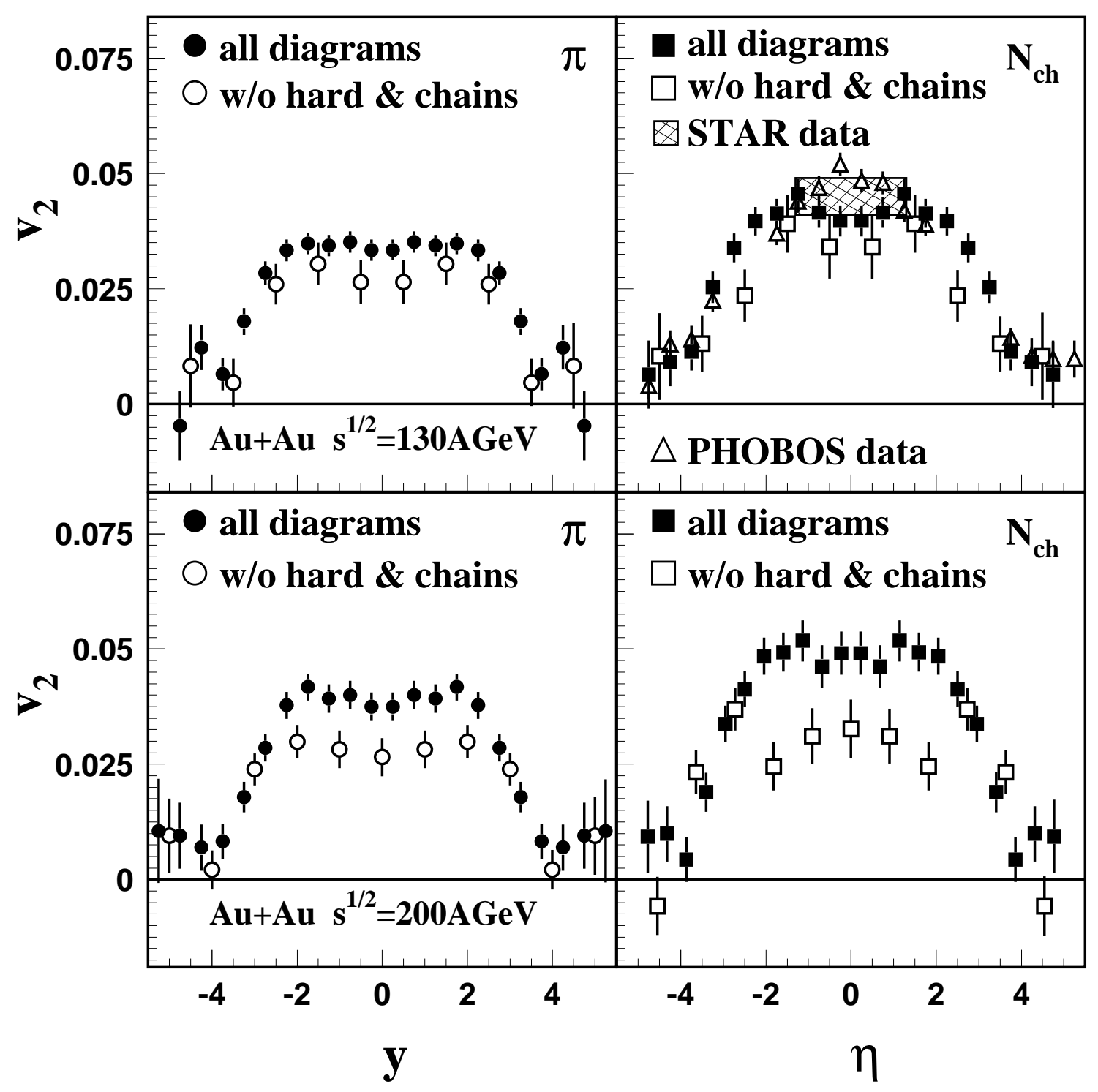

Fig. 2. Elliptic flow of pions (solid circles) and charged particles (solid squares) as a function of rapidity $y$ and pseudorapidity $\eta$ in minimum bias $\mathrm{Au}+\mathrm{Au}$ collisions in the QGSM at $\sqrt{s}=130 A \mathrm{GeV}$ (upper panels) and $200 A \mathrm{GeV}$ (lower panels). Open circles and squares show the elliptic flow in the model version without the hard processes and multi-chain diagrams. Hatched area indicates the data measured by the STAR Collaboration [17], open triangles denote the preliminary results of the PHOBOS Collaboration [35]. 


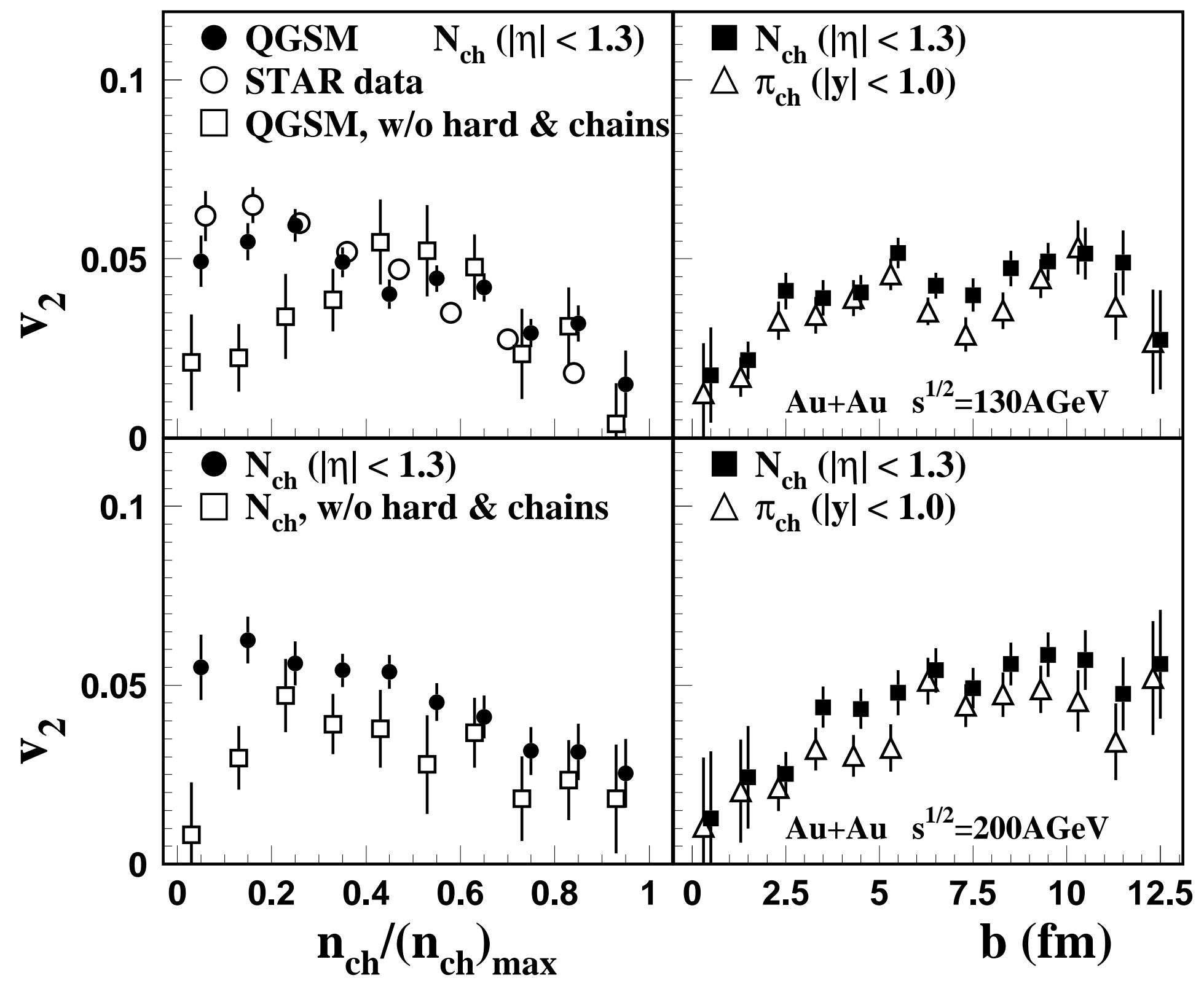

Fig. 3. Elliptic flow of charged particles (solid circles) with $|\eta|<1.3$ as a function of the ratio $n_{c h} /\left(n_{c h}\right)_{\max }$ (left panels) and the impact parameter $b$ (right panels) in minimum bias $\mathrm{Au}+\mathrm{Au}$ collisions at $\sqrt{s}=130 \mathrm{~A} \mathrm{GeV}$ (upper row) and $200 \mathrm{~A}$ $\mathrm{GeV}$ (lower row). The flow of charged pions with $|y|<1$ in the model is shown by open triangles, open circles denote the experimental data from Ref.[17]. Open squares indicate the elliptic flow in the model version without the hard processes and multi-chain diagrams. 


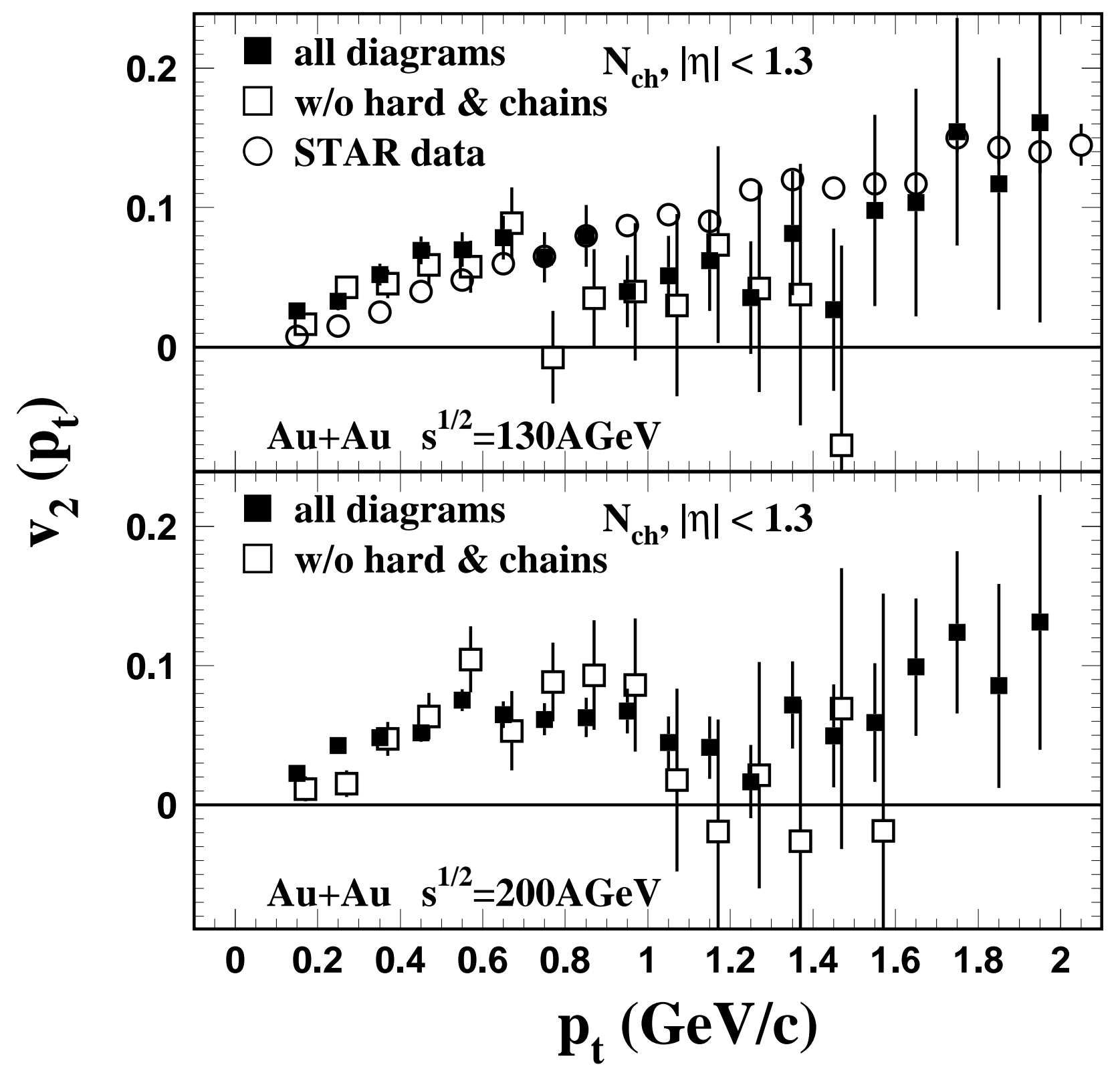

Fig. 4. Transverse momentum dependence of the elliptic flow of charged particles with $|\eta|<1.3$ in minimum bias $\mathrm{Au}+\mathrm{Au}$ collisions at $130 \mathrm{~A} \mathrm{GeV}$ and $200 \mathrm{~A} \mathrm{GeV}$. Data from Ref.[17] are shown by open squares. 